\title{
Philosophiques
}

\section{Claude Lévesque, Dissonance. Nietzsche à la limite du langage, Hurtubise HMH, 1988, 183 pages.}

\section{Marc Turgeon}

Volume 18, numéro 1, printemps 1991

URI : https://id.erudit.org/iderudit/027144ar

DOI : https://doi.org/10.7202/027144ar

Aller au sommaire du numéro

Éditeur(s)

Société de philosophie du Québec

ISSN

0316-2923 (imprimé)

1492-1391 (numérique)

Découvrir la revue

Citer cet article

Turgeon, M. (1991). Claude Lévesque, Dissonance. Nietzsche à la limite du langage, Hurtubise HMH, 1988, 183 pages. Philosophiques, 18(1), 169-194.

https://doi.org/10.7202/027144ar

Ce document est protégé par la loi sur le droit d'auteur. L'utilisation des services d'Érudit (y compris la reproduction) est assujettie à sa politique d'utilisation que vous pouvez consulter en ligne.

https://apropos.erudit.org/fr/usagers/politique-dutilisation/ 


\title{
ÉTUDE CRITIQUE
}

CLAUDE LÉVESQUE, Dissonance. Nietzscbe d la limite du langage, Hurtubise HMH, 1988, 183 pages.

\author{
par Marc Turgeon
}

Le livre de Claude Lévesque nous offre une lecture de Nietzsche dont le propos est de coordonner les thèmes nietzschéens du langage, de la tragédie et de la musique dans le but d'en dégager la philosophie: entendons par ce mot de philosophie une perspective, une position et une traduction de certains thèmes d'un vocabulaire dans un autre. Présentée ainsi la chose peut sembler un peu crue, voire réductrice. C'est pourtant du noud de toute entreprise philosophique qu'il s'agit : quand une telle traduction est achevée, quand une série de métaphores et de définitions en avale une autre, reste-t-il quelque chose? La philosophie peut-elle légitimement aspirer à la vérité, en montrant du doigt les restes des autres, pour enfin les expliquer, ou n'est-elle qu'une suite plus ou moins continue de reprises et de thématisations successives se déployant dans un espace narratif, faisant signe vers le réel mais ne se l'appropriant que métaphoriquement? Il semble que ce soit là la leçon que l'A. nous invite à retenir de l'œuvre de Nietzsche et il faudra se demander si, quand la philosophie est mise à nue et se révèle n'être qu'une hypostase par laquelle on tente de faire le pont entre des états mentaux et le réel afin de donner à ceux-ci un point d'appui, il reste autre chose que le bruit du miroir qui se brise.

Un premier volet de la lecture de l'A. doit être considéré comme un tel effort de traduction. C'est celui qui porte sur la critique nietzschéenne de la rationalité et du langage. Selon l'A., l'essentiel échappe au langage et au concept, l'immédiat ne se laisse approcher qu'indirectement. Le mot exclut * tout rapport immédiat et tout contact empirique ", il " ne fait jamais que suggérer, pointant aveuglément vers un au-delà du langage* (17). Le langage n'est pas une surface paisible et lumineuse d'expression: il se déploie au contraire dans la possibilité de l'excès, car l'homme * est fondamentalement un être soumis à la tension * (19), tension dont la tonalité est essentiellement tragique, porteuse d'une * force incantatoire dont les effets mimétiques et ensorcellants sont immédiats * (20). C'est à cette composante non rationnelle du langage que la * science * résiste en délimitant tout, en * cherchant à dépouiller le monde de son caractère terrifiant * (20). Nietzsche oppose à cela une vision du langage dans laquelle celui-ci est avant tout « langage du désir et des sentiments - (24). C'est ce qu'enseigne la tragédie grecque dans sa forme dionysiaque en conservant au langage sa tonalité lyrique et en reconnaissant en lui * la suprême merveille musicale de la nature * (24). Les mots, formés dans «la béance terrifiante de la bouche qui crie... 
cessent d'être des signes..., ils ne sont plus définis par ce qu'ils veulent dire, mais par le travail qu'ils accomplissent... (26-27). Cet effroi, dimension première du langage, trouve selon l'A. sa confirmation dans les recherches de la psychanalyse sur la parole et l'inconscient: il faut * parler pour parler, à la limite de l'impossibilité de parler, en laissant surgir ce que le langage habituel refoule et frappe d'interdit... (afin) de jouir de la parole et dans la parole, celle-ci ayant alors l'intensité et la souveraineté de la passion * (40).

Voici posées les balises du parcours que l'A. nous propose de La naissance de la tragédie : langage, cri, musicalité, vérité. La figure de Dionysos est fondamentale dans cette constellation car Nietzsche recourre systématiquement à celle-ci, en tant que principe instable et impur, indécidable, afin de valider son refus de l'idée d'une origine pure et transparente du sens, en consonnance avec l'être et la nature. En lieu et place d'une telle origine, Nietzsche rencontre le monde dionysiaque « de la terreur et de la démesure, le monde de la contradiction et de la dissonance..., un déchirement et un cri intolérable que le discours de la raison tente par tous les moyens d'étouffer * (47). Dans cet espace qui s'ouvre à la réflexion du généalogiste, là où la question de l'origine est suspendue, nous sommes contraints de nous arrêter à la musique qui devient, à la place de la recherche du sens, la voie royale de la pensée. C'est en elle, en effet, que se fait entendre * cette dissonance qui constitue l'essence intime des choses * (51). Pour l'A., il * en est du langage comme de la musique : sous le coup de l'émotion et de la passion, le langage se nie comme discursivité et se donne comme parole intensive et accentuée, allant, dans le risque absolu de ce qui est hors sens, jusqu'à la limite proprement inouïe du cri * (53). C'est la possibilité musicale du cri qui ouvre le langage: * toujours divisé, toujours pluriel, toujours discordant et contradictoire * (57), le cri est dissonance, *l'intervalle qui introduit le silence dans la pensée et, plus radicalement, ... qui la rend possible* (54).

La méditation sur la musique et la dissonance devient donc le prétexte à une mise en cause de la culture, c'est-à-dire une mise en cause de la forme de la pensée qui préside à la définition de la philosophie, travail qui se fait, dans l'œuvre de Nietzsche, par le biais de l'élaboration de la complémentarité entre Apollon et Dionysos. Le premier incarne une parole ordonnée à une finalité, une parole rationnelle comme celle que l'on trouve dans l'œuvre d'Euripide; le second inspire davantage l'œuvre d'Eschyle, plus près du culte dionysiaque, conviant une parole * trébuchante, inadéquate, ... aveugle par rapport à elle-même * (101). L'un incarne une vision optimiste, l'autre une vision tragique; le premier tend au platonisme et fonde une rationalité triomphante, le second *exprime ce qu'il y a de douloureux et d'horrible dans l'existence* (102). Quelque chose échappe à la dialectique optimiste du platonisme, un supplément qui est précisément l'objet du *langage de la Dionysie, une manière de dire, sans le réduire, ce reste inabordable, indéchiffrable... (107). Le reste dont il s'agit nous est donné dans le parlé-chanté et le cri du lyrisme tragique. Puisque le langage est la plus haute forme musicale de la nature, le cri est donc la plus haute manifestation du langage : «L'ivresse du sentiment réussit à élever le langage jusqu'à la pure sonorité, jusqu'au cri, jusqu'à la dissonance* (115). Le 
dithyrambe n'est pas ordonné à un message mais à l'affectivité. Dès que celle-ci survient, * la musicalité du langage passe au premier plan alors que le message recule et s'assombrit en se disloquant* (121). Affectivité et harmonie vont de pair et fondent la notion de dissonance. Celle-ci définit non seulement ce qui est le plus musical dans la musique mais aussi ce qui est le plus langagier dans le langage. I.'harmonie est à la musique ce que le cri est au langage : « le cri est la dimension musicale du langage, à la limite du langage, et I'harmonie est la dimension purement musicale de la musique, à la limite de la musique et du bruit * (130). À l'opposé de la consonnance qui, comme la sérénité apollinienne, est un effet de surface, l'harmonie est liée à la dissonance, elle est «l'espacement nécessaire pour que joue pleinement la fascination pour tout ce qui est extrême et impossible* (133). Cet impossible nous condamnerait au nihilisme si la fascination pour l'absurde n'était rien d'autre que cela : fascination pour la perte, l'obscur, le refus de la clarté apollinienne. Mais cette dimension que Nietzsche voit en Apollon n'est pas première : c'est le cri du satyre qui l'est, en même temps qu'il se trouve voilé de la lumière d'Apollon. Il ne faut pas nier ce dernier au profit de Dionysos, mais retrouver celle-ci pour mieux comprendre celui-là; ne plus chercher l'origine dans les termes d'une contradiction, mais l'essence ou langage dans la tension de ces deux forces. Entre l'optimisme béat et le pessimisme le plus absolu naît la pensée tragique, * une pensée audacieuse qui se tient à la limite de la pensée, en équilibre instable entre la beauté et la vérité, et qui cache, sous le voile antique du mythe, une vérité d'abîme * (174).

À quoi nous engage cette pensée tragique, qu'est-ce qui vient après Nietzsche, quelle sorte de philosophie est encore possible? L'A. répond à ces questions de deux manières : en nous disant qu'il faut libérer le cri de l'emprise de la raison et en ajoutant qu'il faut tenir compte dans la discussion de la symbolisation, de la dimension rhétorique de la communication. Le monde de la tragédie, écrit-il, est le * monde intermédiaire du vraisemblable - ce monde qui est aussi celui de la rhétorique " (178). Dans l'espace abyssal du cri *le langage retrouve son épaisseur sonore, sa matérialité différentielle, sa puissance de transposition et de séduction, bref sa dimension lyrique et rhétorique * (28). Les pensées sont des actions, elles sont les signes d'un jeu et d'un combat des émotions. Le langage est métaphorique et les concepts portent en eux l'oubli de leur nature symbolique: « on commence par tracer un cercle magique autour de certains éléments du langage... et l'on rejette à l'extérieur de cette enceinte sacrée les éléments non manipulables... Tout ce qui apparaît comme informe, confus, inarticulé, inanalysable, est rejeté, comme le cri, l'accent, la plainte, l'intonation, le ton. Bref, ce qui est de l'ordre de l'expérience, de la vie, de l'affectivité, de la gestualité, de l'écriture et du style est refoulé dans la marge...* (35-36). Dans la perspective ouverte par Nietzsche, toutefois, la connaissance est indissociable de la méta. phore qui la construit, il n'y a pour lui aucune « naturalité non-rhétorique du langage » (30), le symbole est toujours détaché de la réalité, n'en est jamais qu'une traduction plus ou moins agréée. La réflexion de Nietzsche sur la musique et la tragédie apparaît ainsi comme une longue hyperbole pour démontrer que l'on ne peut * penser un discours sans énonciateur, un énoncé sans énonciation, une dénotation sans connotation, ... (un) 
langage sans fantasmes * (38). Les mots * participent à la constitution proprement rhétorique du langage et sont dès l'origine, et par "nature", des figures de rhétorique*, ils composent * un ensemble de figures rhétoriques dont l'étrange articulation caractérise nettement le style dionysiaque (121) et qui accueillent les forces de «non-parole* de la tragédie.

L'A. semble hésiter devant cette question : ces forces de non-parole, qualifiées de rhétoriques, à quel genre de philosophie nous convientelles? Une possibilité, riche et souvent pratiquée, consiste dans le ressassement des métaphores nietzschéennes. Ce retour sur le texte de Nietzsche peut prendre deux formes : celle du commentaire érudit et celle du commentaire littéraire, et l'A. s'adonne aux deux. Si, toutefois, le premier trouve sa justification dans le débat général sur l'histoire des idées, le second pose un problème nietzschéen : ce dernier dénonce en effet l'oubli de la métaphore dans l'élaboration conceptuelle et veut remplacer l'idéal de pure pensée par l'idée que les systèmes philosophiques sont marqués d'une "tonalité, une teinte personnelle qui nous permettent de reconstituer la figure du philosophe * (cité, 72); il dénonce le cercle magique dans lequel Socrate enferme la pensée, excluant tout ce qui est rhétorique, mais comment, si l'on veut filer quelque chose à partir de ses écrits, échapper au cercle magique de leurs métaphores ? Comment récupérer ce qui relève de la rhétorique autrement qu'en écrivant sur un mode littéraire à partir des métaphores de Nietzsche et de la reprise de ces métaphores dans des ceuvres comme celles de Blanchot ou Derrida? N'y a-t-il pas quelque chose de paradoxal (que Blanchot, à tout le moins, a su mesurer) à vouloir maintenir dans un lieu d'énonciation universitaire un effort de pensée qui invite à fonder l'écriture en clle-même plutôt que dans la pensée et l'académie ? N'est-ce pas là le paradoxe de la généalogie ? Ne pouvoir (la philosophie) être son propre objet sans être liée à ce ressassement éternel ? Ne pouvoir (la philosophie) se déployer comme discours sans se nier comme entreprise de conceptualisation et ne plus pouvoir se dire autrement que sous la forme de la casuistique, étude cas par cas des paroles, des écrits, des circonstances ou alors, sur un tout autre plan, comme cuvre d'art? On aurait souhaité, sans doute pour que ces impasses semblent moins incontournables, que l'A. s'arrête davantage au «Qui ?" nietzschéen. Là où notre culture philosophique fait défaut, si l'on peut dire, là où la rhétorique lui échappe et lui pose problème, c'est dans la question du sujet. La formule philosophique la plus inédite est sans doute, pour le dire à la manière de Nietzsche : "Qui * - Pas moi ! * Formule par laquelle le sujet s'absente et fait de son discours un discours sur l'autre. Si l'on veut comprendre l'insistance de l'A. à déplorer l'oubli de la métaphore, il faut sans doute revenir avec lui à La Naissance de la tragédie pour y voir, à la suite de Nietzsche, la naissance de la philosophie (la naissance d'un oubli) et poser la question : qui parle ici ? qui souffre, désire, jubile? Quelle est sa volonté?

Attaché qu'il est à discuter du langage en dansant autour de la figure de Dionysos, figure de l'ambiguïté et de l'indécision, on peut comprendre que l'A. trouve son équilibre sur la corde raide en oscillant entre dire les choses en termes de "rhétorique * ou de * forces de la non-parole *, entre des métaphores qui cernent le domaine de l'indicible dans la 
parole et celles qui tentent de le mimer: $\propto$ Sous l'emprise d'une force démoniaque... il se produit une telle excitation des sentiments et de l'imagination que l'esprit du compositeur et, aussi bien, de l'auditeur, est emporté au-delà de lui-mêne, de sorte que les frontières de l'individualité s'abolissent en quelque manière * (22)... * Il se pourrait que cette autre voix, cette voix étrangère qui se fait entendre dans le dithyrambe dionysiaque... soit effectivement étrangère au moi et à tout moi, qu'elle soit à la fois la voix la plus secrète, la plus singulière, la plus personnelle et la voix de personne, une voix radicalement impersonnelle, inappropriable * (73). Cet indicible, cette dissonance ne peuvent être qu'entendus, ils ne sont là que pour l'oreille de l'autre. Mais cette oreille, le tiers si important en rhétorique comme en psychanalyse, et qui pourrait ouvrir ici sur une thématisation de l'altérité, débouche en fait sur d'autres métaphores de l'impossible à dire : «l'élévation de l'affect introduit dans le labyrinthe de l'oreille quelque chose d'insensé et d'absurde qui la tympanise et l'ouvre à ce qui est infigurable, imperceptible et inimaginable * (88). Cette inquiétante étrangeté à laquelle nous convie l'expérience tragique, nous oublions trop souvent que, pour inappropriable qu'elle soit, nous n'en tentons pas moins - comme en témoigne la psychanalyse - de lui donner une forme dicible et d'assurer par là une certaine (re)construction du sujet. Comme on oublie aussi que le récit que fait Nietzsche de la naissance de la tragédie ne fait pas que critiquer l'Être, mais fonde également un discours sur la culture. Pourquoi, si l'A. accepte que, dans * la poésie lyrique et le drame antique, il n'est pas question de jouir de la musique d'une façon isolée, mais toujours au contraire en relation avec le culte*, et que, en * son essence le langage est rhétorique et... rythmique * (108), pourquoi renchérir en ajoutant que le cri est *l'analogon du chaos originaire * (109), que la langue est une * symbolique des forces démoniaques *, qu'elle est * dans sa structure même, une langue chantante * (112), si ce n'est l'effet d'une fascination pour la langue nietzschéenne? Loin de nous l'idée de déprécier cette fascination mais celle-ci, pour admirable qu'elle soit, donne au lecteur l'envie de percer le miroir et d'y échapper. Cette célébration du texte de Nietzsche, et c'est là un ton qui convient néanmoins et que Nietzsche commande chez plusieurs de ses lecteurs, peut-être peut-elle s'expliquer du fait que * ce n'est que lorsqu'elle est chantée que la pensée atteint l'apogée de son action et qu'elle se constitue véritablement comme symbole compréhensible de l'indéchiffrable volonté * (114). Mais alors - La pensée se fait poésie "(114). Comment recevoir une telle injonction? La position paradoxale de l'A. est une invite à chanter mais en même temps une résistance à le faire, à larguer les amarres, à abandonner l'université, à aller vers la culture plutôt que vers l'institution, à se faire cuvre plutôt que critique, comme Nietzsche a choisi de le faire. On peut rappeler ici le curieux jugement de G. Colli qui écrivait (Après Nietzsche, Éd. de l'Éclat), à propos du désir de Nietzsche qu'une a action dépasse les limites du papier et de l'encrier *, qu'il était déplorable que celui-ci n'ait * perçu que rarement l'étroitesse de l'agir par la littérature * (25). Avait-il le choix, se voulant généalogiste, c'est-à-dire sans lieu d'où faire originer la pensée, et musicien, c'est-à-dire sans autre parole que celle de la création? La question du langage converge certes, chez Nietzsche, vers la figure de Dionysos, mais la revendication par celui-ci de cette 
figure à son propre compte invite à revenir sur sa critique des liens dénaturés de la culture et de l'université dans laquelle il exige que nous engagions notre destin, notre détresse et notre chance dans la pensée si nous voulons « espérer aboutir à quelque résultat * (70).

L'A. n'a choisi ni la poésie ni l'exil du poète. Il semble avoir préféré, à bon droit, de parcourir Nietzsche, d'en être la voix autant que l'oreille. Est-ce nietzschéen, pourrait-on demander malicieusement? Ou est-ce un combat discret pour le droit de la philosophie à la métaphore? Le droit à une autre culture philosophique, peut-être pas moins académique mais, du moins plus humble et plus gaie à la fois devant les tâches de la pensée, moins policée par le désir ou le fantasme de la vérité, une pensée pouvant en appeler davantage à l'autre ? Nous sommes tentés de chercher les réponses à ces questions dans les citations qui ouvrent et ferment le livre de Claude Lévesque : «Derrière le discours parle le refus de discourir, comme derrière la philosophie parlerait le refus de philosopher: parole non parlante, violente, se dérobant, ne disant rien et tout à coup criant (Blanchot); «On aperçoit toujours dans les écrits d'un solitaire une sorte d'écho du désert, quelque chose qui rappelle le murmure et la circonspection farouche de la solitude; même dans ses paroles les plus violentes, et jusque dans ses cris, résonne encore une manière nouvelle, plus dangereuse, de se taire et d'imposer silence à sa parole* (Nietzsche).

Département de philosopbie Collège de Saint-Laurent 